\title{
Pre-hospital alarm activation for STEMI patients undergoing primary percutaneous coronary intervention in the era of transradial procedures
}

Fabio Rigamonti ${ }^{1}$, Baris Gencer ${ }^{1}$, Florian Rey ${ }^{1}$, Jawad Chaara ${ }^{1}$, Elena Tessitore ${ }^{1}$, Sholan Bunwaree $^{1}$, Pascal Meier ${ }^{1}$, Stéphane Noble ${ }^{1}$, Olivier Grosgurin ${ }^{2,3}$, Robert Larribeau ${ }^{3}$, Stephan Windecker $^{4}$, Federico Carbone ${ }^{5}$, Fabrizio Montecucco ${ }^{5,6}$, Nicolas Rodondi ${ }^{7}$, Christian M. Matter ${ }^{8,9}$, Thomas F. Luscher ${ }^{8,9}$, François Mach $^{1}$, Marco Roffi ${ }^{1}$

${ }^{1}$ Division of Cardiology, Department of Medical Specialties, Geneva University Hospitals, 4 rue Gabrielle-Perret-Gentil, 1211 Geneva, Switzerland. Fabio.rigamonti@hcuge.ch; baris.gencer@hcuge.ch; florian.rey@hcuge.ch; _ jawad.chaara@hcuge.ch; elena.tessitore@hcuge.ch; sholanbunwaree@yahoo.co.uk; pascal.meier@hcuge.ch; stephane.noble@hcuge.ch; francois.mach@hcuge.ch; marco.roffi@hcuge.ch

${ }^{2}$ Division of General Internal Medicine, Department of Medical Specialties, Geneva University Hospitals, 4 rue Gabrielle-Perret-Gentil, 1211 Geneva, Switzerland. olivier.grosgurin@hcuge.ch

${ }^{3}$ Division of Emergency Medicine, Department of Medical Specialties, Geneva University Hospitals, 4 rue Gabrielle-Perret-Gentil, 1211 Geneva, Switzerland. robert.larribau@ @cuge.ch 4 Division of Cardiology, Bern University Hospital, 3010 Bern, Switzerland. stephan.windecker@insel.ch

${ }^{5}$ First Clinic of Internal Medicine, Department of Internal Medicine, University of Genoa. 6 viale Benedetto XV, 16132 Genoa, Italy. Federico.carbone@edu.unige.it; Fabrizio.montecucco@unige.it

6 IRCCS AOU San Martino - IST, 10 largo Benzi, 16132 Genoa, Italy. Fabrizio.montecucco@unige.it 
${ }^{7}$ Department of General Internal Medicine, Inselspital, Bern University Hospital, 3010 Bern, Switzerland.nicolas.rodondi@insel.ch

${ }^{8}$ Department of Cardiology, University Heart Center, University Hospital Zurich, Rämistrasse 100, 8091 Zürich, Switzerland.christian.matter@uzh.ch

${ }^{9}$ Center for Molecular Cardiology, University of Zurich, Wagistrasse 12, 8952 Schlieren, Switzerland.cardiotfl@gmx.ch

Corresponding Author: Fabio Rigamonti, MD, Division of Cardiology, Department of Medical Specialties, Geneva University Hospital, 4 rue Gabrielle-Perret-Gentil, 1205 Geneva 14, Switzerland, Tel: +41 $22 \quad 37272$ 00, Fax: +41 $22 \quad 37272$ 29, E-mail: fabio.rigamonti@hcuge.ch

Running title: pre-hospital alarm and STEMI. 


\section{Abstract}

Background: Transradial access (TRA) improves outcome compared with trans-femoral access for the management of patients with acute coronary syndromes. In this setting, it is unknown whether the activation of a pre-hospital alarm system (PHAS) confers additional benefit for the prognosis of patients with ST-elevation myocardial infarction (STEMI).

Materials and methods: We retrospectively analyzed a cohort of patients with a first STEMI who underwent a primary percutaneous coronary intervention (PPCI) at a single center within a prospective cohort of acute coronary syndrome patients (SPUM-ACS). TRA was used in $85 \%$ of patients. We assessed how PHAS ( $n=165)$ vs. no-PHAS $(n=166)$ activation was associated with the composite outcome of all-cause mortality and recurrence of myocardial infarction (MI) at 1-year follow-up. As secondary outcomes, the individual clinical endpoints were separately assessed for association. Results: Compared with no-PHAS patients, patients in the PHAS group were predominantly women, and presented more frequently with dyslipidemia and cardiac arrest. A significant reduction in the composite outcome of all-cause mortality and recurrent MI at 1-year was observed in the PHAS group, compared with noPHAS (3.6\% vs $8.5 \%, \mathrm{p}=0.027)$. When adjusted for age, sex and resuscitation status, PHAS activation remained associated with decreased all-cause mortality and recurrent MI (HR: 0.36 [95\% CI: 0.13-0.95]; $\mathrm{p}=0.040)$.

Conclusions: This study suggests that the benefit of PHAS activation in STEMI patients undergoing PPCI persists also in the era of TRA.

Keywords: STEMI, primary PCI, pre-hospital alarm system, transradial access. 


\section{Introduction}

Guidelines from the European Society of Cardiology (ESC) and the American College of Cardiology Foundation/American Heart Association (ACCF/AHA) recommend as class I immediate reperfusion therapy for ST-segment elevation myocardial infarction (STEMI) (1, 2].The goal is to perform a primary percutaneous intervention (PCI) in $<90 \mathrm{~min}$ and $\leq 60 \mathrm{~min}$ if the patient directly arrives to a PCI-capable hospital. Key to achieve those goals are common management protocols shared within regional networks and emergency medical systems (EMSs). As a consequence, EMSs and hospital networks were set up to bypass the Emergency Department (ED) and reach the recommended time to PPCI. Some examples of successful setups are illustrated by the Mayo Clinic STEMI protocol [3], the Minnesota regional system of care [4], the Vienna STEMI network [5], the Service d'Aide Médicale Urgente (SAMU) network (based on a unique emergency call number in France) [6], or the Geneva STEMI alarm [7]. A previous study from our research group described a significant improvement in the door-to-balloon time (DTBT) through the STEMI alarm system [7]. The benefits of a prehospital alarm system (PHAS) to reduce the delays of and improve the outcomes in the setting of PPCI using a trans-femoral approach (TFA) are well documented [8]. Recently, the superiority of the transradial approach (TRA) in patients with acute coronary syndromes (ACS) undergoing PCI was demonstrated in terms of major bleeding, vascular complications, ischemic events as well as all-cause mortality [9]. However, these results derived from high volume centers with experimented operators in TRA. It is less clear if those benefits are confirmed in moderate volume centers with a recent switch from TFA to TRA and less experimented operators, in particular in the high challenging situation of STEMI. In this context, the technically complexity of TRA might also raise concerns about length of interventions, fluoroscopy duration and crossover rates. Despite these limitations, guidelines now recommend TRA as the method of choice for vascular access in ACS patients 
[10]. Therefore, considering the global positive clinical impact of TRA for PPCI, we investigated whether PHAS activation maintained this benefit in a single center with operators experimented in TRA.

\section{Methods}

Study population and design

We conducted a single-center nested study within a prospective cohort, the Special Program University Medicine Acute Coronary Syndromes and Inflammation (SPUM-ACS), and evaluated consecutive patients treated with PPCI for STEMI in at the Geneva University Hospital between January 2010 and December 2013. The SPUM-ACS (NCT01000701) was established by 4 Swiss University Hospitals (Bern, Geneva, Lausanne and Zurich) to prospectively recruit and analyze a real-world cohort of 5'751 consecutive patients with a diagnosis of ACS who underwent coronary angiography [11]. Participants were then followed up by telephone by a trained study nurse at 30 days post-STEMI, and again by a follow-up visit at one-year. If patients were unable to attend the clinic visit, follow-up was performed by one of the following options (by order of importance): (1) phone call, (2) postal mail or email, (3) through family members, or (4) via primary care physician or cardiologist.

For this analysis, we included consecutive patients with a first STEMI, defined as the concomitant presence of symptoms compatible with myocardial ischemia and the three following criteria: (a) ECG ischemic changes such as persistent or dynamic ST-segment elevation or new left bundle branch block; (b) evidence of positive conventional or highsensitive troponin by local laboratory reference values with a rise and/or fall in enzyme levels; (c) documented culprit lesion of coronary artery at coronary angiography. Exclusion criteria included previous documented myocardial infarction (MI), severe physical disability, inability or unwillingness to give consent, or a life expectancy of less than one year for non-cardiac 
reasons. Patients transferred via ambulance or helicopter from France were excluded from this analysis, due to the impossibility to collect detailed pre-hospital delays. The protocol was approved by the local institutional review board and the Ethical Committee; all participants gave written informed consent.

\section{Data Collection}

Of the eligible 584 patients treated with PPCI for STEMI at Geneva University Hospital, we identified 331 patients enrolled in the SPUM-ACS who met the inclusion criteria and were included in this analysis. SPUM-ACS collects, among other information, demographic data, comorbidities, treatment at admission and discharge, hemodynamic data, as well as clinical outcomes at 30 days and one year. Based on the pre-hospital medical report in the hospital's electronic medical records database, patients were divided into two groups according to whether or not they had been admitted with PHAS activation. The door-time (DT) was the time of ED admission for patients admitted with no PHAS, while DTBT was the entry time in the cardiac catheterization unit in the presence of PHAS. For all patients, additional information, such as procedure time, radiation exposition, quantity of contrast dye used, as well as type of arterial approach (TRA or TFA) were extracted from the catheterization laboratory electronic database. All PPCI procedures were reviewed by two experienced interventional cardiologists to gather records of the balloon time (BT), as well as the time of guidewire passage of the culprit lesion.

\section{PPCI description}

The local standard pre-PPCI therapy in case of STEMI includes intravenous administration of $250 \mathrm{mg}$ aspirin, a $5000 \mathrm{IU}$ bolus of unfractionated heparin and an oral loading dose of prasugrel $(60 \mathrm{mg})$ or clopidogrel $(600 \mathrm{mg})$ either in the ambulance or in the emergency 
department (ED). The modified Allen test using pulse oximetry and plethysmography was routinely performed before puncturing the radial artery to ensure sufficient hand perfusion in case of radial occlusion [12]. PPCI was performed using mainly 6Fr guiding catheters. Additional unfractionated heparin was administered according to the activated clotting time. After procedure completion the sheath was removed immediately, and a compression bracelet (TR-Band, Terumo, Japan) was installed for 3 hours with a standardized progressive deflation protocol. In case of TFA, PPCI was performed using 6Fr guiding catheters. Procedural success, was defined as TIMI flow III in the culprit vessel at the end of the TRA or TFA procedure. At the end of the procedure, an ilio-femoral angiogram was obtained and a vascular closure device (AngioSeal, St. Jude Medical, St. Paul, Minnesota; Starclose or Perclose, Abbott Vascular Devices, Redwood City, California) was deployed upon confirmation of suitable vascular anatomy and a proper vascular entry site. When the use of such devices was contraindicated, the sheath was removed in a delayed fashion and manual compression was performed until satisfactory haemostasis was achieved. This was followed by placement of a pressure dressing for 6 hours. In the absence of contraindication, double antiplatelet therapy was prescribed for one year.

\section{Study Endpoints}

The primary endpoint of the study was the composite outcome of all-cause mortality and recurrence of MI at 1-year follow-up. As secondary endpoints all-cause mortality and recurrence of MI at 1-year follow-up were separately assessed. As part of the SPUM-ACS protocol, all events were adjudicated by a panel of three independent certified cardiologists blinded to the management strategy.

\section{Statistical analyses}


Statistical analyses were performed using the Stata statistical software, release 13.1® (Stata Corporation, College Station, TX, USA) and Statview (SAS institute Inc, Cary, NC, USA). The PHAS group was compared to no-PHAS activation using Pearson's chi-square test or Fisher's exact test (when appropriate) for the comparison of qualitative variables. The MannWhitney nonparametric test (the normality assumption of the variables' distribution in both groups was violated) was used for comparisons of continuous variables. For continuous variables, results were expressed as medians (interquartile range [IQR]). Kaplan-Meier survival analysis was performed to estimate cumulative event rate follow-up and to calculate the corresponding risk difference according to the study groups. Specifically, given the relative limited sample size, risk difference was calculated using Cox's F test [13]. Finally, the effect of PHAS activation on the risk of the endpoints was estimated by Cox proportional hazard models and expressed with hazard ratios (HR) and 95\% CI. Multivariate analysis was performed considering PHAS activation, age, sex and resuscitation state in order to obtain adjusted estimates of the HRs and to identify factors independently associated with the outcomes. Variables were expressed as medians (IQR). Values with $\mathrm{p}<0.05$ (two-tailed) were considered significant.

\section{Results}

\section{Patients' characteristics}

Baseline demographic, clinical and biochemical characteristics of the no-PHAS $(n=165)$ and PHAS $(n=166)$ activation groups are shown in Table 1 . The proportion of men in the noPHAS group was higher compared to the PHAS group $(82.4 \%$ vs. $73.5 \%, \mathrm{p}=0.050)$. The study groups had similar baseline comorbidities, except for the following parameters that were more frequently encountered in the PHAS group compared to the no-PHAS activation group: history of dyslipidaemia ( $43.6 \%$ vs. $56.0 \%$; $\mathrm{p}=0.024)$, chest pain duration $<24 \mathrm{~h}$ prior to 
hospitalization $(89.1 \%$ vs. $78.3 \%$; p=0.011), and PPCI cardiac arrest $(10.8 \%$ vs. $4.9 \%$, $\mathrm{p}=0.043$ ) (Table 1). Medical therapy on admission, at discharge (Table 2) and at 1-year follow-up (Table 3) was comparable across both groups.

\section{PHAS reduces DTBT delay, but does not influence percutaneous PCI procedure}

As expected, PHAS activation markedly reduced DTBT compared to no-PHAS (Table 4). This beneficial reduction could be demonstrated in terms of median DTBT time $<90$ minutes as well as for the proportion of patients with DTBT $\leq 60$ minutes (Table 4). With regard to the vascular access site, PHAS activation reduced DTBT for both TRA and TFA procedures, compared to no-PHAS (Table 4). The technical characteristics of PPCI were similar in both study groups (Table 5). TRA was performed in $87.9 \%$ no-PAHS and $83.7 \%$ PHAS patients. The rate of PPCI failure was of $0.6 \%$ (1 patient) in the no-PHAS group and $1.2 \%$ ( 2 patients) in the PHAS activation group. Procedural success was achieved in $94.6 \%$ no-PHAS patients and $94.0 \%$ PHAS patients. Only one patient in the overall cohort underwent urgent coronary artery bypass surgery. In the majority of patients enrolled, a stent was implanted $(93.9 \%$ and 95.2\% in the no-PHAS and PHAS groups, respectively). No differences were found for median time of fluoroscopy (11.3 vs.11.7; $\mathrm{p}=0.945)$ and median quantity of contrast utilized (170 ml vs. $170 \mathrm{ml}$; $\mathrm{p}=0.862$ ) (Table 5). The conversion rate from TRA to TFA was similar in both groups: $1.2 \%$ and $2.4 \%$ in the non-PHAS and PHAS groups, respectively $(\mathrm{p}=0.180)$.

PHAS improves the composite outcome of all-cause mortality and recurrent MI rates at 1year follow-up

Based on a Kaplan-Meyer analysis, the composite primary endpoint of all-cause mortality and recurrent $\mathrm{MI}$ at one year was $8.5 \%(14 / 165)$ in no-PHAS patients and $3.6 \%(6 / 166)$ in the PHAS group ( $\mathrm{p}=0.027$, Cox's F test, Figure 1). The independent predictors of all-cause 
mortality and recurrent MI are listed in Table 6. In particular, PHAS activation was associated with a significant reduction in events (adjusted HR: 0.36 [95\% CI: 0.13-0.95]; p=0.040) (Table 6). When clinical outcomes were separately assessed, prediction by PHAS became non-significant (Table 7). Due to low number of clinical events (when outcomes were separately assessed), we had to concomitantly reduce in the analysis the number of co-variates (including only age, sex and PHAS). Considering the low statistical power of this analysis, no definite conclusion can be formulated on separate outcomes.

\section{DISCUSSION}

The main finding of this analysis is that, in the setting of PPCI performed mainly by TRA, PHAS activation is associated with a reduction in all-cause mortality or recurrent MI at 1 year in STEMI patients, with a marked reduction of DTBT compared to no-PHAS activation. This finding, in addition to an optimal medical management over 1 year post-PPCI, underlines the importance of PHAS activation even in the era of improved PPCI outcomes resulting from the switch from TFA to TRA.

The management of STEMI patients has substantially evolved over the past five decades, resulting in a major decline in all-cause mortality, recurrent MI, and heart failure rates [14]. In order to reduce DTBT and myocardial injury, current guidelines make a number of recommendations: that PPCI be performed within 90 minutes of the first medical contact (FMC); procedures be centralized in high-volume centers; and dedicated pre-hospital management protocols including PHAS activation be developed [15]. Successful examples of timeliness optimization between regional non-PCI-hospitals and PCI high-volume centers since the year 2000 are provided by the Mayo clinic STEMI-alarm system that enabled a DTBT $<90 \%$ to be achieved in $75 \%$ of patients; the Minnesota regional system $[3,4]$, that led to a median DTBT of $95 \mathrm{~min}$ (79\% of patients with DTBT <120 min) for community 
hospitals situated up to 60 miles from a PCI center resulting in a 1-year all-cause mortality of 7.2\%; the Vienna STEMI network that enabled coordination between 5 high-volume PCI centers and led to a mean DTBT of $81 \mathrm{~min}$, with a consequent drop of in-hospital mortality to $9.5 \% ;^{5}$ and the Geneva alarm system that led to a reduction of DTBT from 109 to 76 min after introducing the PHAS activation system, with $66 \%$ of patients reperfused $<90$ min [7]. Conversely, a recent analysis of the American Cath-PCI Registry showed no improvement for in-hospital mortality despite a reduction in the DTBT from 83 to 67 minutes [16]. Vascular PPCI access may have played a critical role in this setting, since US registries enrolled mainly patients treated by TFA $[17,18]$, and studies on pre-hospital protocols and regional networks paid little attention to this technical aspect despite its apparent crucial impact on outcomes. The MATRIX trial that compared TRA with TFA for ACS in 8404 patients, showed a reduction of net adverse clinical events (9.8\% vs $11.7 \%$; RR of 0.83 , C.I: $0.73-0.96)$ at 30 days follow-up to be associated with TRA and a reduction in all-cause mortality (1.6\% vs 2.2\%; RR of 0.72, C.I: 0.53-0.99) [9]. A meta-analysis including MATRIX confirmed these findings, with a significant reduction in major adverse cardiovascular events and all-cause mortality associated with TRA. For this reason, recent guidelines have given the highest degree of recommendation (Class I A) to the TRA approach in ACS patients [10]. As the guidelines recommend that centers treating ACS patients implement a transition from TFA to TRA, the challenge will be for the younger generation of interventionists to maintain TFA skills, which remain critical for procedures like intra-aortic counter pulsation implantation, structural heart procedures, or peripheral vascular interventions. As limitations of our study, only patients with a first STEMI who were enrolled in the SPUM-ACS study (56\% of all eligible STEMI patients admitted during that time at our institution) were included in this analysis. Although a selection bias cannot be excluded, this is counterbalanced by the fact that in the SPUM-ACS cohort, all events were independently adjudicated. The follow-up time was 
relatively short, reducing the possibility to detect long-term effects of PHAS. Another limitation could be that this was a single-center retrospective non-randomized observational study with a modest number of patients enrolled. Therefore, this approach and design did not allow additional sub-analysis in order to assess the procedure-related results in different age categories. In conclusion, PHAS activation was independently associated with a reduction of all-cause mortality and recurrent MI at 1-year follow-up in patients with a first STEMI treated by PPCI mainly via TRA.

Conflict of interest statement: none to be declared.

\section{Acknowledgments}

The work was supported by the Swiss National Science Foundation (SPUM 33CM30-124112 and SPUM 33CM30-140 336, Inflammation and acute coronary syndromes (ACS) - Novel strategies for prevention and clinical management). Special gratitude is expressed to Aliki Buhayer (Prism Scientific Sàrl) for medical writing support. 


\section{LEARNING POINTS}

Transradial access improves outcome compared with trans-femoral access for the management of patients with acute coronary syndromes, but it is unknown whether the activation of a pre-hospital alarm system confers additional benefit.

In the setting of primary percutaneous coronary intervention performed mainly by transradial access, pre-hospital alarm activation was independently associated with better clinical outcomes at one-year follow up in patients with ST-elevation myocardial infarction.

Similarly to previous studies, pre-hospital alarm activation was also able to reduce the timeto-treatment in these patients. 


\section{References}

[1] Task Force on the management of ST-segment elevation acute myocardial infarction of the European Society of Cardiology (ESC), Steg PG, James SK, Atar D, Badano LP, Blömstrom-Lundqvist C, Borger MA, et al. ESC Guidelines for the management of acute myocardial infarction in patients presenting with ST-segment elevation. Eur Heart J. 2012;33:2569-619.

[2] American College of Emergency Physicians; Society for Cardiovascular Angiography and Interventions, O'Gara PT, Kushner FG, Ascheim DD, Casey DE Jr, Chung MK, de Lemos JA, et al. 2013 ACCF/AHA guideline for the management of ST-elevation myocardial infarction: a report of the American College of Cardiology Foundation/American Heart Association Task Force on Practice Guidelines. J Am Coll Cardiol. 2013;61:e78-140.

[3] Ting HH, Rihal CS, Gersh BJ, Haro LH, Bjerke CM, Lennon RJ, et al. Regional systems of care to optimize timeliness of reperfusion therapy for ST-elevation myocardial infarction: the Mayo Clinic STEMI Protocol. Circulation. 2007;116:729-36.

[4] Henry TD, Sharkey SW, Burke MN, Chavez IJ, Graham KJ, Henry CR, et al. A regional system to provide timely access to percutaneous coronary intervention for STelevation myocardial infarction. Circulation. 2007;116:721-8.

[5] Kalla K, Christ G, Karnik R, Malzer R, Norman G, Prachar H, et al.; Vienna STEMI Registry Group. Implementation of guidelines improves the standard of care: the Viennese registry on reperfusion strategies in ST-elevation myocardial infarction (Vienna STEMI registry). Circulation. 2006;113:2398-405.

[6] Cambou JP, Simon T, Mulak G, Bataille V, Danchin N. The French registry of Acute ST elevation or non-ST-elevation Myocardial Infarction (FAST-MI): study design and baseline characteristics. Arch Mal Coeur Vaiss. 2007;100:524-34. 
[7] Grosgurin O, Plojoux J, Keller PF, Niquille M, N'koulou R, Mach F, et al. Prehospital emergency physician activation of interventional cardiology team reduces door-to-balloon time in ST-elevation myocardial infarction. Swiss Med Wkly. 2010;140:228-32.

[8] Nallamothu BK, Bradley EH, Krumholz HM. Time to treatment in primary percutaneous coronary intervention. N Engl J Med. 2007;357:1631-38.

[9] Valgimigli M, Gagnor A, Calabró P, Frigoli E, Leonardi S, Zaro T, et al.; MATRIX Investigators. Radial versus femoral access in patients with acute coronary syndromes undergoing invasive management: a randomised multicentre trial. Lancet. 2015;385:2465-76.

[10] Roffi M, Patrono C, Collet JP, Mueller C, Valgimigli M, Andreotti F, et al. 2015 ESC Guidelines for the management of acute coronary syndromes in patients presenting without persistent ST-segment elevation: Task Force for the Management of Acute Coronary Syndromes in Patients Presenting without Persistent ST-Segment Elevation of the European Society of Cardiology (ESC). Eur Heart J. 2016;37:267-315.

[11] Gencer B, Montecucco F, Nanchen D, Carbone F, Klingenberg R, Vuilleumier N, et al. Prognostic value of PCSK9 levels in patients with acute coronary syndromes. Eur Heart J. 2016;37:546-53.

[12] Barbeau GR, Arsenault F, Dugas L, Simard S, Larivière MM. Evaluation of the ulnopalmar arterial arches with pulse oximetry and plethysmography: comparison with the Allen's test in 1010 patients. Am Heart J. 2004;147:489-93.

[13] Regal D. The F test with time-censored exponential data. Biometrika. 1980;67:479-81.

[14] Windecker S, Bax JJ, Myat A, Stone GW, Marber MS. Future treatment strategies in ST-segment elevation myocardial infarction. Lancet. 2013;382:644-57.

[15] Stone GW, Dixon SR, Grines CL, Cox DA, Webb JG, Brodie BR, et al. Predictors of infarct size after primary coronary angioplasty in acute myocardial infarction from pooled analysis from four contemporary trials. Am J Cardiol. 2007;100:1370-5. 
[16] Menees DS, Peterson ED, Wang Y, Curtis JP, Messenger JC, Rumsfeld JS, et al. Door-to-balloon time and mortality among patients undergoing primary PCI. N Engl J Med. 2013;369:901-9.

[17] Iqbal MB, Khamis R, Ilsley C, Mikhail G, Crake T, Firoozi S, et al.; London Heart Attack Centre Group Investigators. Time-trend analyses of bleeding and mortality after primary percutaneous coronary intervention during out of working hours versus in-working hours: an observational study of 11466 patients. Circ Cardiovasc Interv. 2015;8: doi: 10.1161/CIRCINTERVENTIONS.114.002206.

[18] Howe MJ, Seth M, Riba A, Hanzel G, Zainea M, Gurm HS. Underutilization of Radial Access in Patients Undergoing Percutaneous Coronary Intervention for ST-SegmentElevation Myocardial Infarction: Insights From the Blue Cross Blue Shield of Michigan Cardiovascular Consortium. Circ Cardiovasc Interv. 2015;8. doi: 10.1161/CIRCINTERVENTIONS.114.002036. 


\section{Figure legends}

Figure 1. Composite primary outcome (all-cause mortality and re-infarction rate) according to PHAS or no-PHAS activation. Kaplan-Meier time-to-events plot for the composite outcome (all-cause mortality and re-infarction rate) at 1-year follow- up. 
Table 1. Baseline characteristics of patients admitted for STEMI.

\begin{tabular}{|c|c|c|c|}
\hline & no-PHAS* $(n=165)$ & PHAS $(n=166)$ & $p$-value \\
\hline \multicolumn{4}{|l|}{ Demographic } \\
\hline Men, no. (\%) & $136(82.4)$ & $122(73.5)$ & 0.050 \\
\hline Age, yr. & $60.9(51.9-69.7)$ & $60.85(50.1-69.0)$ & 0.795 \\
\hline Body mass index, $\mathrm{kg} / \mathrm{m}^{2}$ & $26.2(24.1-29.3)$ & $26.1(24.4-29.1)$ & 0.743 \\
\hline History of diabetes, no. (\%) & $26(15.8)$ & $22(13.3)$ & 0.518 \\
\hline History of dyslipidemia, no. (\%) & $72(43.6)$ & $93(56.0)$ & 0.024 \\
\hline History of hypertension, no. (\%) & $75(45.5)$ & $76(45.8)$ & 0.952 \\
\hline History of CAD ${ }^{++}$, no. (\%) & $49(29.7)$ & $35(21.1)$ & 0.072 \\
\hline $\mathrm{LVEF}^{\dagger}<40 \%$ at ad ${ }^{\circ}$, no $(\%)$ & $39(23.6)$ & $55(33.1)$ & 0.080 \\
\hline History of PVD ${ }^{*}$ no. $(\%)$ & $4(2.4)$ & $5(3.0)$ & 0.742 \\
\hline History of malignancy, no. (\%) & $15(9.1)$ & $10(6.0)$ & 0.298 \\
\hline History of lung disease, no. (\%) & $2(1.2)$ & $5(3.0)$ & 0.255 \\
\hline History of $\mathrm{GI}^{\&}$ bleeding, no. $(\%)$ & $4(2.4)$ & $2(1.2)$ & 0.406 \\
\hline History of inflammatory disease, $(\%)$ & $1(0.6)$ & $1(0.6)$ & 0.997 \\
\hline History of valvular disease, no. (\%) & $0(0.0)$ & $1(0.6)$ & 0.318 \\
\hline History of stroke, no. (\%) & $4(2.4)$ & $3(1.8)$ & 0.696 \\
\hline Tobacco use: current, no. (\%) & $74(44.9)$ & $76(45.8)$ & 0.864 \\
\hline Previous PCI", no. $(\%)$ & $6(3.6)$ & $4(2.4)$ & 0.514 \\
\hline \multicolumn{4}{|l|}{ Clinical presentation } \\
\hline \multicolumn{4}{|l|}{ Chest pain duration prior $\mathrm{PPCI}^{\Delta}$} \\
\hline$<24 \mathrm{~h}$, no. $(\%)$ & $147(89.1)$ & $130(78.3)$ & 0.011 \\
\hline $24-72 \mathrm{~h}$, no. $(\%)$ & $11(6.7)$ & $18(10.8)$ & 0.179 \\
\hline$>72 \mathrm{~h}$, no. $(\%)$ & $7(4.2)$ & $17(10.2)$ & 0.034 \\
\hline ECG without STEMI ${ }^{\#}$ criteria & $8(4.9)$ & $4(2.4)$ & 0.235 \\
\hline \multicolumn{4}{|l|}{ Killip classification at ad. ${ }^{\&}$} \\
\hline I, no. $(\%)$ & $148(89.7)$ & $138(83.1)$ & 0.082 \\
\hline II, no. $(\%)$ & $6(3.6)$ & $12(7.2)$ & 0.150 \\
\hline III, no. (\%) & $2(1.2)$ & $2(1.2)$ & 0.995 \\
\hline IV, no. (\%) & $7(4.2)$ & $13(7.8)$ & 0.166 \\
\hline SBP ${ }^{\| l l}$ at ad., mmHg. & $119(103-135)$ & $120(105-132)$ & 0.972 \\
\hline $\mathrm{DBP}^{* *}$ at ad., $\mathrm{mmHg}$. & $74(63-82)$ & $75(64-84)$ & 0.476 \\
\hline Pre-PPCI CA ${ }^{\# \#, ~ n o . ~(\%) ~}$ & $8(4.9)$ & $18(10.8)$ & 0.043 \\
\hline Vasopressor therapy, no. (\%) & $7(4.2)$ & $5(3.0)$ & 0.557 \\
\hline \multicolumn{4}{|l|}{ Biochemical } \\
\hline Troponin I. at ad., mg/l & $0.54(0.15-7.2)$ & $0.52(0.09-2.9)$ & 0.177 \\
\hline Peak troponin I. , mg/l & $50.0(15.3-94.0)$ & $64.2(23.6-97.8)$ & 0.304 \\
\hline $\mathrm{Ck}^{\dagger \dagger}$ value at ad., $\mathrm{U} / \mathrm{l}$ & $298.5(133-685)$ & 216(119-662) & 0.245 \\
\hline Peak ck value., U/l & $1533(611-2827)$ & $1801(872-3455)$ & 0.115 \\
\hline $\mathrm{Hb}^{\circ}$ at ad., gr/l & $146(131-156)$ & $142(131-153)$ & 0.184 \\
\hline Creatinine at ad., umol/1 & $83(70-95)$ & $81(66-96)$ & 0.442 \\
\hline
\end{tabular}

Data are expressed as median (interquartile range) or number (no.), percentages (\%). Two-tailed Fisher's exact tests for dichotomous variables, larger Chi-squared test for ${ }^{+}$Independence, and Mann-Whitney-U tests for continuous variables, were utilized as appropriate.

* PHAS: Pre-hospital alarm activation.

${ }^{++}$CAD: Coronary artery disease.

${ }^{\dagger}$ LVEF: Left ventricular ejection fraction.

* PVD: Peripheral vascular disease.

$\S$ GI: Gastrointestinal.

" PCI: Percutaneous coronary intervention.

${ }^{++}$: 1 missing patient for this variable in the PHAS group.

\# STEMI: ST-segment elevation myocardial infarction.

${ }^{\Delta}$ : PPCI: Primary percutaneous coronary intervention.

${ }^{I \mid l}$ SBP: systolic blood pressure.

${ }^{* *}$ DBP: diastolic blood pressure.

${ }^{\# \#} \mathrm{CA}$ : cardiac arrest. 
${ }^{\dagger} \mathrm{CK}$ : creatin-kinase.

${ }^{\circ} \mathrm{Hb}$ : Haemoglobin.

${ }^{\circ}$ ad. : admission.

$\stackrel{\&}{ }$ : 2 missing values in the no-PHAS group. 
Table 2. Medications listed at first medical contact and at prescribed discharge for patients admitted for STEMI.

\begin{tabular}{|c|c|c|c|}
\hline & no-PHAS $^{*}(\mathrm{n}=165)$ & PHAS $(n=166)$ & $p$-value \\
\hline \multicolumn{4}{|l|}{ Medications at FMC } \\
\hline Aspirin, no. (\%) & $27(16.4)$ & $18(10.8)$ & 0.143 \\
\hline Clopidogrel, no. (\%) & $3(1.8)$ & $4(2.4)$ & 0.708 \\
\hline Oral anticoagulation, no. (\%) & $3(1.8)$ & $3(1.8)$ & 0.994 \\
\hline $\mathrm{ACE}^{\S}$ therapy, no. $(\%)$ & $12(7.3)$ & $14(8.4)$ & 0.683 \\
\hline AT-II" therapy, no. (\%) & $34(20.6)$ & $31(18.7)$ & 0.678 \\
\hline B-blocker therapy, no. (\%) & $21(12.7)$ & $18(10.8)$ & 0.595 \\
\hline Calcium antagonists, no. (\%) & $19(11.5)$ & $13(7.8)$ & 0.257 \\
\hline Amiodarone, no. $(\%)$ & $2(1.2)$ & $0(0.0)$ & 0.155 \\
\hline Digoxin, no. (\%) & $1(0.6)$ & $2(1.2)$ & 0.565 \\
\hline Antiarrythmics others, no. (\%) & $2(1.2)$ & $1(0.6)$ & 0.558 \\
\hline Nitrate, no. $(\%)$ & $1(0.6)$ & $1(0.6)$ & 0.997 \\
\hline Diuretic therapy, no. (\%) & $19(11.5)$ & $15(9.0)$ & 0.458 \\
\hline Insulin therapy, no. (\%) & $6(3.6)$ & $6(3.6)$ & 0.991 \\
\hline NSAID ${ }^{\ddagger}$, no. $(\%)$ & $3(1.8)$ & $6(3.6)$ & 0.315 \\
\hline Proton pump inhibitors, no. (\%) & $12(7.3)$ & $13(7.8)$ & 0.848 \\
\hline Statin, no. $(\%)$ & $25(15.2)$ & $28(16.9)$ & 0.670 \\
\hline \multicolumn{4}{|l|}{ Medications at discharge $^{\dagger}$} \\
\hline Aspirin, no. (\%) & $161(97.6)$ & $164(98.8)$ & 0.406 \\
\hline Clopidogrel, no. (\%) & $66(40.0)$ & $59(35.5)$ & 0.403 \\
\hline Prasugrel, no. (\%) & $77(46.7)$ & $95(57.2)$ & 0.055 \\
\hline Ticagrelor, no. (\%) & $15(9.7)$ & $8(4.2)$ & 0.126 \\
\hline Oral anticoagulation, no. (\%) & $30(18.2)$ & $20(12.0)$ & 0.120 \\
\hline ACE therapy, no. (\%) & $136(82.4)$ & $147(88.6)$ & 0.113 \\
\hline ATI-I therapy, no. (\%) & $20(12.1)$ & $13(7.8)$ & 0.193 \\
\hline B-blocker therapy, no. (\%) & $154(93.3)$ & $158(95.2)$ & 0.470 \\
\hline Calcium antagonists, no. (\%) & $3(1.8)$ & $4(2.4)$ & 0.708 \\
\hline Amiodarone, no. $(\%)$ & $6(3.6)$ & $7(4.2)$ & 0.786 \\
\hline Digoxin, no. (\%) & $0(0.0)$ & $1(0.6)$ & 0.318 \\
\hline Antiarrythmics others, no. (\%) & $3(1.8)$ & $2(1.2)$ & 0.647 \\
\hline Nitrate, no. $(\%)$ & $9(5.5)$ & $13(7.8)$ & 0.401 \\
\hline Diuretic therapy, no. $(\%)$ & $29(17.6)$ & $26(15.7)$ & 0.640 \\
\hline Insulin therapy, no. (\%) & $7(4.2)$ & $5(3.0)$ & 0.549 \\
\hline $\mathrm{NSAID}^{\dagger}$, no. $(\%)$ & $1(0.6)$ & $1(0.6)$ & 0.997 \\
\hline Proton pump inhibitors, no. (\%) & $31(18.8)$ & $35(21.1)$ & 0.601 \\
\hline IST $^{\circ}$, no. $(\%)$ & $4(2.4)$ & $3(1.8)$ & 0.696 \\
\hline Hormone therapy, no. (\%) & $9(5.5)$ & $7(4.2)$ & 0.600 \\
\hline Antidepressant, no. (\%) & $5(3.0)$ & $9(5.4)$ & 0.280 \\
\hline Statin, no. $(\%)$ & $162(98.1)$ & $164(98.8)$ & 0.647 \\
\hline
\end{tabular}

Data are expressed as number (no.), percentages (\%). Two-tailed Fisher's exact tests for dichotomous variables for Independence were employed.

${ }^{\vartheta}$ FMC: First medical contact

*PHAS: Pre-hospital alarm system.

IST: Immunosuppressive therapy.

: NSAID: Non steroidal anti-inflammatory drug.

t: 3 missing therapy report in the no-PHAS group and 2 missing therapy reports in the PHAS group.

§: ACE: Angiotensin converting enzyme.

" : AT-II: Angiotensin receptor II antagonist. 
Table 3. Medications taken by patients admitted for STEMI at one-year follow-up.

\begin{tabular}{|c|c|c|c|}
\hline & no-PHAS $^{*}(n=165)$ & PHAS $(n=166)$ & $p$-value \\
\hline \multicolumn{4}{|l|}{ Medications at 1 year follow-up ${ }^{\dagger}$} \\
\hline Aspirin, no. (\%) & $147(89.1)$ & $157(94.6)$ & 0.068 \\
\hline Clopidogrel, no. (\%) & $48(29.1)$ & $43(25.9)$ & 0.516 \\
\hline Prasugrel, no. (\%) & $55(33.3)$ & $67(40.4)$ & 0.185 \\
\hline Ticagrelor, no. (\%) & $11(6.7)$ & $5(3.0)$ & 0.121 \\
\hline Oral anticoagulation, no. (\%) & $6(3.6)$ & $7(4.2)$ & 0.786 \\
\hline $\mathrm{ACE}^{\S}$ therapy, no. $(\%)$ & $98(59.4)$ & $95(57.2)$ & 0.690 \\
\hline ATI-I" therapy, no. (\%) & $36(21.8)$ & $35(21.1)$ & 0.871 \\
\hline B-blocker therapy, no. (\%) & $122(73.9)$ & $129(77.7)$ & 0.423 \\
\hline Calcium antagonists, no. (\%) & $10(6.1)$ & $11(6.6)$ & 0.833 \\
\hline Amiodarone, no. (\%) & $5(3.0)$ & $3(1.8)$ & 0.469 \\
\hline Digoxin, no. (\%) & $1(0.6)$ & $0(0.0)$ & 0.315 \\
\hline Antiarrythmics others, no. (\%) & $2(1.2)$ & $1(0.6)$ & 0.558 \\
\hline Nitrate, no. $(\%)$ & $4(2.4)$ & $6(3.6)$ & 0.527 \\
\hline Diuretic therapy, no. $(\%)$ & $22(13.3)$ & $26(15.7)$ & 0.547 \\
\hline Insulin therapy, no. (\%) & $6(3.6)$ & $4(2.4)$ & 0.514 \\
\hline $\mathrm{NSAID}^{\ddagger}$, no. $(\%)$ & $2(1.2)$ & $4(0.60)$ & 0.414 \\
\hline Proton pump inhibitors, no. (\%) & $24(14.6)$ & $28(16.7)$ & 0.562 \\
\hline $\mathrm{IST}^{\circ}$, no. $(\%)$ & $3(1.8)$ & $3(1.8)$ & 0.994 \\
\hline Hormone therapy, no. (\%) & $7(4.2)$ & $9(5.4)$ & 0.617 \\
\hline Antidepressants, no. (\%) & $14(8.5)$ & $9(5.4)$ & 0.273 \\
\hline Statin, no. (\%) & $142(86.1)$ & $149(89.8)$ & 0.302 \\
\hline
\end{tabular}

Data are expressed as number (no.),percentages (\%). Two-tailed Fisher's exact tests for dichotomous variables for independence were employed.

*PHAS: Pre-hospital alarm system.

$\dagger: 14$ missing therapy reports in the no-PHAS group and 7 in the PHAS group.

IST: Immunosuppressive therapy.

*NSAID: Non-steroidal anti-inflammatory drug.

${ }^{\S}$ ACE: Angiotensin converting enzyme.

" AT-II: Angiotensin receptor II antagonist. 
Table 4. Door-to-balloon time (DTBT) for patients admitted for STEMI.

\begin{tabular}{lccr}
\hline & No-PHAS ${ }^{\dagger}(\mathbf{n = 1 6 5})$ & PHAS $(\mathbf{n = 1 6 6})$ & $\boldsymbol{p}$-value \\
\hline DTBT & & & \\
\hline DTBT $^{*} \min$ & $85(46-171)$ & $54(35-72)$ & $<\mathbf{0 . 0 0 1}$ \\
DTBT $<90$ min, no. $(\%)$ & $83(50.3)$ & $147(88.6)$ & $<\mathbf{0 . 0 0 1}$ \\
DTBT $\leq 60$ min, no. $(\%)$ & $56(33.9)$ & $96(57.8)$ & $<\mathbf{0 . 0 0 1}$ \\
DTBT min with TRA & $85(46-186)$ & $55(35-72)$ & $<\mathbf{0 . 0 0 1}$ \\
DTBT min with TFA & $103(43-153)$ & $46(33-66)$ & $\mathbf{0 . 0 0 5}$ \\
\hline
\end{tabular}

Data are expressed as median interquartile range or number (no.), percentages (\%).

*DTBT: door-to-balloon time.

${ }^{\dagger}$ PHAS: pre-hospital alarm system. 
Table 5. PPCI procedure-related characteristics for STEMI.

\begin{tabular}{|c|c|c|c|}
\hline & No-PHAS $(n=165)$ & PHAS $(n=166)$ & $p$-value \\
\hline \multicolumn{4}{|l|}{ Characteristic of PPCI } \\
\hline Radial access ${ }^{\#}$, n. (\%) & $145(87.9)$ & $139(83.7)$ & 0.529 \\
\hline $\mathrm{CABG}^{*}$, n. $(\%)$ & $0(0.0)$ & $1(0.6)$ & 0.318 \\
\hline $\mathrm{IABP}^{\dagger}$, n. $(\%)$ & $9(5.5)$ & $9(5.4)$ & 1.000 \\
\hline Stent implantation, n. (\%) & $155(93.9)$ & $158(95.2)$ & 0.619 \\
\hline DES $^{\ddagger}$ implantation in culprit, n. (\%) & $103(62.4)$ & $114(68.7)$ & 0.231 \\
\hline $\mathrm{BMS}^{\S}$ implantation in culprit, n. (\%) & $52(31.5)$ & $44(26.5)$ & 0.315 \\
\hline TIMI flow III post-PPCI ${ }^{\circ 0^{\circ}}, \mathrm{n} .(\%)$ & $156(94.6)$ & $156(94.0)$ & 0.767 \\
\hline Conversion $^{* *}$ from TRA to TFA, n. (\%) & $2(1.2)$ & $4(2.4)$ & 0.423 \\
\hline Time of fluoroscopy, min. & $11.3(7.77-18.58)$ & $11.7(8.40-16.20)$ & 0.945 \\
\hline Time of fluoroscopy with TRA, min. & $10.8(7.77-19.43)$ & $11.8(8.40-17.42)$ & 0.770 \\
\hline Time of fluoroscopy with TFA, min. & $14.3(9.12-21.24)$ & $10.7(7.03-14.46)$ & 0.132 \\
\hline Total amount of dye, $\mathrm{ml}$. & $170(130-210)$ & $170(130-220)$ & 0.862 \\
\hline Total amount of dye with TRA, ml. & $170(130-210)$ & $170(130-220)$ & 0.862 \\
\hline Total amount of dye with TFA, ml. & $160(135-205)$ & $166(150-200)$ & 0.873 \\
\hline
\end{tabular}

Data are expressed as median (interquartile range) or number (no.), percentages (\%). Two-tailed Fisher's exact tests for dichotomous variables, and Mann-Whitney-U tests for continuous variables, were utilized as appropriate.

PPCI: Primary percutaneous coronary intervention.

${ }^{*} \mathrm{CABG}$ : Coronary-artery by-pass graft surgery.

IIABP: Intra-aortic balloon pump. 1 missing report for this variable in the PHAS group.

${ }^{\#}$ For the radial access variable: 1 missing report in the non-PHAS and 3 missing values in the PHAS group.

${ }^{*}$ DES: Drug eluting stent. Ten missing reports for this variable in the no-PHAS group and 8 in the PHAS group.

${ }^{\S}$ BMS: Bare metal stent. Ten missing reports for this variable in the no-PHAS group and 8 in the PHAS group.

4 missing reports for this variable in the no-PHAS group and 4 in the PHAS group.

** 1 missing report for this variable in the non-PHAS group and 2 in the PHAS group. 
Table 6. Univariate and multivariate analysis of age, sex, resuscitation status and PHAS with regard to clinical primary and secondary outcomes (all-cause mortality and myocardial re-infarction) at one year.

\begin{tabular}{lcccc}
\hline Independent variables & \multicolumn{2}{c}{ Univariate analysis } & \multicolumn{2}{c}{ Multivariate analysis } \\
& $\mathrm{HR}^{+}\left(95 \% \mathrm{CI}^{\dagger}\right)$ & $p$-value & HR $(95 \%$ CI $)$ & $p$-value \\
\hline Age & \multicolumn{1}{c}{ Composite outcome of death and myocardial re-infarction } & \\
Sex & $1.07(1.03-1.11)$ & $<\mathbf{0 . 0 0 1}$ & $1.07(1.02-1.11)$ & $\mathbf{0 . 0 0 2}$ \\
PHAS & $2.45(1.00-6.00)$ & 0.050 & $1.61(0.61-4.22)$ & 0.335 \\
Resuscitation status $^{*}$ & $0.42(0.16-1.09)$ & 0.074 & $0.36(0.13-0.95)$ & $\mathbf{0 . 0 4 0}$ \\
\hline
\end{tabular}

* PHAS: pre-hospital alarm system.

${ }^{\dagger}$ CI: Confidence Interval.

$\$$ HR: Hazard ratio. 
Table 7. Univariate and multivariate analysis of age, sex and PHAS with regard to secondary outcomes (all-cause death or myocardial re-infarction) at one year.

\begin{tabular}{lcccc}
\hline Independent variables & \multicolumn{2}{c}{ Univariate analysis } & \multicolumn{2}{c}{ Multivariate analysis } \\
& HR $^{\ddagger}\left(95 \% \mathrm{CI}^{\dagger}\right)$ & $p$-value & HR $(95 \%$ CI $)$ & $p$-value \\
\hline Age & $1.10(1.03-1.17)$ & $\mathbf{0 . 0 0 3}$ & $1.09(1.02-1.16)$ & $\mathbf{0 . 0 1 5}$ \\
Sex & $4.52(1.21-16.84)$ & $\mathbf{0 . 0 2 4}$ & $2.52(0.61-10.46)$ & 0.204 \\
PHAS & $0.50(0.13-1.99)$ & 0.324 & $0.38(0.94-1.58)$ & 0.184 \\
\hline & & Myocardial re-infarction & $1.05(1.00-1.10)$ & $\mathbf{0 . 0 4 9}$ \\
Age & $1.05(1.00-1.10)$ & $\mathbf{0 . 0 4 9}$ & $0.86(0.21-3.44)$ & 0.826 \\
Sex & $1.18(0.32-4.37)$ & 0.800 & $0.32(0.85-1.19)$ & 0.088 \\
\hline
\end{tabular}

* PHAS: pre-hospital alarm system.

${ }^{\dagger} \mathrm{CI}$ : Confidence Interval.

${ }^{\ddagger}$ HR: Hazard ratio. 
Figure 1

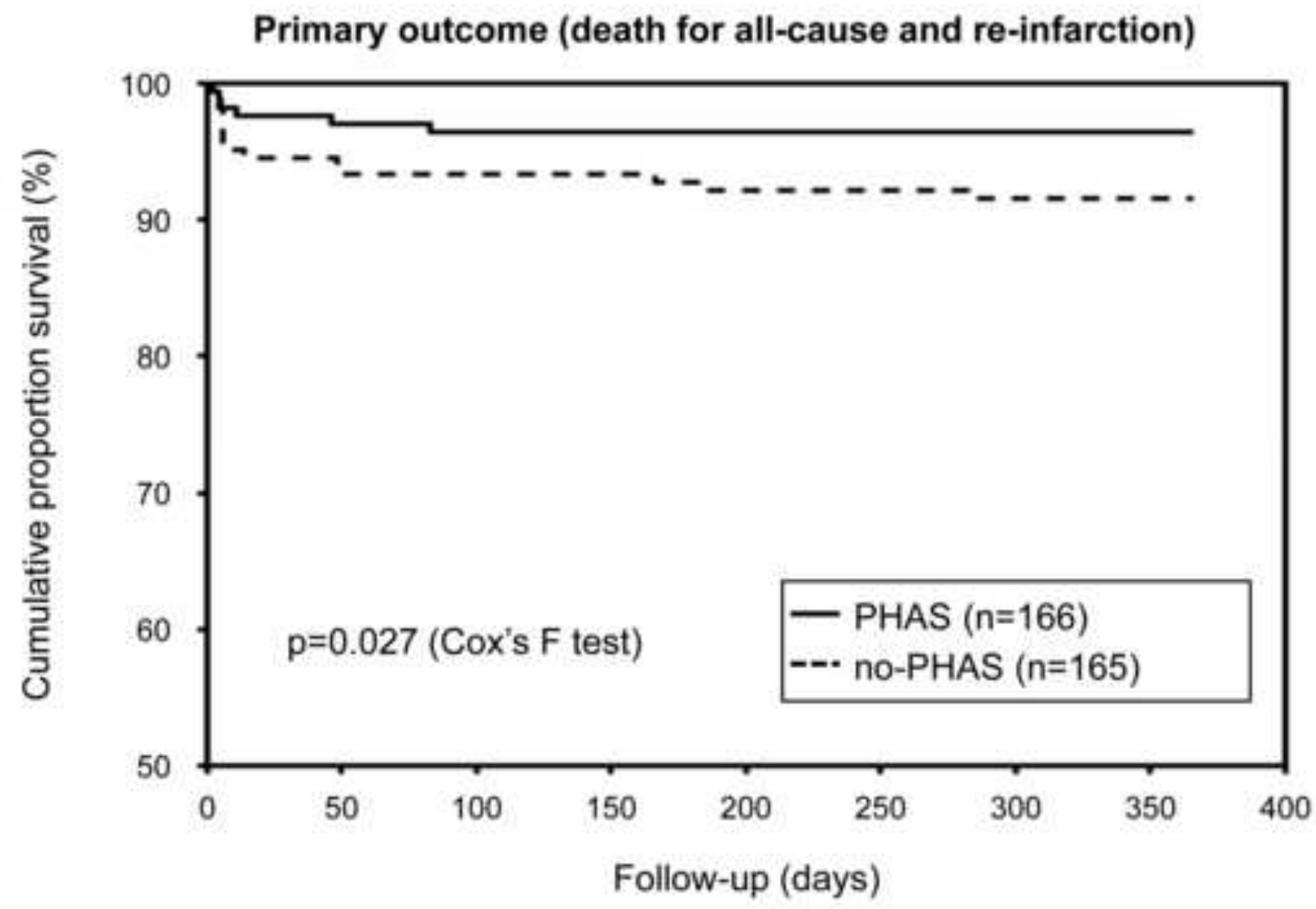

